Research Article

\title{
Realization of the Ultrasonic Motor Speed Control System Based on H-Bridge
}

\author{
Tan Jiawan, Yan Langtao $\mathbb{D}^{\mathrm{D}}$, Liu Yusheng, and Huang Sanshan \\ School of Shipping and Naval Architecture, Chongqing Jiaotong University, Chongqing 400074, China \\ Correspondence should be addressed to Yan Langtao; yanlt023@126.com
}

Received 15 August 2021; Revised 15 September 2021; Accepted 21 October 2021; Published 10 November 2021

Academic Editor: Omar Naifar

Copyright (c) 2021 Tan Jiawan et al. This is an open access article distributed under the Creative Commons Attribution License, which permits unrestricted use, distribution, and reproduction in any medium, provided the original work is properly cited.

In view of the requirement that the speed of rotor speed of ultrasonic motor can be stabilized quickly, combined with the mathematical model of two-phase traveling wave ultrasonic motor, we designed the rotor speed stability control strategy of the two-phase traveling wave ultrasonic motor based on voltage and current double feedback. We simulated, analyzed, and verified the designed strategy using Matlab/Simulink simulation tool. The rationality of the simulation results proves the correctness of the designed control strategy, which lays a theoretical foundation for the production design of two-phase traveling wave ultrasonic motor.

\section{Introduction}

Ultrasonic motors have the advantages [1] of small weight, low speed and large torque, low noise, self-locking when power is off, and no magnetic field interference. Ultrasonic motors have been widely used in research fields such as optical autofocus, medical equipment, robots, instrumentation, and military equipment. There are many control means for ultrasonic motor in different application fields, such as vector control, robust adaptive back-stepping motion control, state feedback control, FPGA-based computed force control, disturbance-observer-based complementary sliding-mode speed control, high-order sliding-mode control, Hermite neural network-based second-order slidingmode control, and sensorless control. Each control method has its own unique advantages. Ultrasonic motor is the core component in many of the previously mentioned fields, and its own function and the performance of the control system will directly affect the development process of the previously mentioned research fields.

For the research of ultrasonic motor control system, the methods often used at present include analytical method, experimental method, and simulation method. Because the analytical method requires a sufficiently complete theoretical knowledge of ultrasonic motors and a comprehensive understanding of the motor control system, the analytical method has greater limitations in the research of ultrasonic control systems. The experimental method requires the establishment of an actual system, and the required experiment can only be made under the premise of a complete control system, so this also leads to the experimental method being inferior to the simulation method in the research of the control system. The simulation method is to establish a complete mathematical or physical model for the ultrasonic motor control system and use the built model to reflect the real behavior of the system. The use of simulation method to study the actual control system is not only economical and safe but also has a good guiding significance for the design and development of new products with better performance. This article aims at stabilizing the speed of the two-phase traveling wave ultrasonic motor, designing corresponding control strategies [2] in combination with the actual circuit, and using simulation methods to verify the rationality and correctness of the design.

\section{Mathematical Model of Two-Phase Traveling Wave Ultrasonic Motor}

2.1. Modeling Assumptions. In order to derive the formula in the modeling process more concisely and the calculation is 
convenient enough, the following idealized condition [3] assumptions are made during the modeling process:

(1) The initial phase angle is ignored

(2) The damping effect of the stator is ignored

(3) The piezoelectric ceramic two-phase polarization zone is completely symmetrical; the voltage amplitudes of corresponding two-phase excitation are also completely equal

(4) Only the constant friction between the stator and the rotor is considered, and the sliding friction is ignored

(5) The contact surface between the stator and the rotor is idealized; that is, the contact surface is just tangent to the vibration waveform of the stator

(6) Regarding the rotor and friction layer as ideal rigid and elastic bodies, the deformation of the rotor surface during operation is not taken into account

(7) The influence of preload on the operating characteristics of ultrasonic motor is ignored

\subsection{Mathematical Model}

\subsubsection{Mathematical Model of Stator Surface Particles}

(1) Displacement Equation of Particle Transverse Vibration on Stator Torus. According to Kirchhoff's circular thin plate theory, on the premise of modeling assumptions, the vibration of particles on the stator torus can be regarded as the superposition of standing waves excited by $L 1$ and $L 2$ twophase driving voltages, and the transverse vibration displacement equation of corresponding particles is as follows:

$$
A(x, t)=A_{0}[\cos k x \operatorname{co\omega } t+\sin k x \sin (\omega t-\alpha)],
$$

where $A_{0}$ represents the transverse vibration amplitude of the particle. The letter $k$ indicates the vibration wave number of elastic wave, and $k=2 \pi / \lambda$; the letter $\lambda$ indicates wavelength. The letter $x$ represents the distance from a point on the straight beam to the origin after the stator is outspread. The letter $\omega$ represents the angular frequency of the drive voltage. The letter $\alpha$ indicates the phase difference between $L 1$ and $L 2$ driving voltages.

If $\alpha= \pm \pi / 2$, formula (1) can be written as

$$
A(x, t)=A_{0}\left[\cos k x \operatorname{co\omega } t+\sin k x \sin \left(\omega t \pm \frac{\pi}{2}\right)\right]=A_{0}(\cos k x \operatorname{co\omega } \omega t \sin k x \sin \omega t)=A_{0} \cos (k x \mp \omega t)
$$

It can be seen from (2) that when the phase difference between $L 1$ and $L 2$ driving voltages is 90 degrees, under the premise of modeling assumption, the vibration mode of particles on the stator torus can be regarded as a traveling wave formed by the superposition of two-phase standing waves, as shown in Figure 1.

It can be seen from Figure 1 that if one phase of the two-phase driving voltage is reversed and the other phase remains unchanged, the phase difference of the two driving voltages changes from the original 90 degrees to -90 degrees after the reverse, the traveling wave formed by the superposition of the two-phase standing waves will change its travel direction, and the rotation direction of the rotor of the ultrasonic motor is reversed from the original direction. In order to simplify the expression form of mathematical equation, only the phase difference of 90 degrees is analyzed in this paper and the analysis process is similar when the phase difference is -90 degrees [4].

(2) Velocity Equation of Particle Vibration on Stator Torus. $P 0$ is set as the position when the elastomer is not bent, and $P$ is the position after bending deformation when $t=0$. The bending angle is $\theta$, and the thickness of piezoelectric vibrator is $h$, as shown in Figure 2.

From Figure 2, the displacement expression of vibration deformation [5] along the thickness direction ( $Z$ - axis direction in Figure 2) from the original point $P 0$ to the deformed point $P$ is as follows:

$$
S_{z}=A(x, t)-\frac{h}{2}(1-\cos \theta)
$$

If the bending angle $\theta$ is small enough, (3) can be rewritten as

$$
S_{z} \approx A(x, t)=A_{0} \cos (k x-\omega t) .
$$

According to formulas (1) and (2), the displacement expression of the particle on the stator torus when it vibrates and deforms in the longitudinal direction ( $x$-axis direction in Figure 2) is as follows:

$$
S_{x}=\frac{k h A_{0}}{2} \sin (k x-\omega t) .
$$

According to formulas (4) and (5), the vibration displacement of stator surface particles in the $x$-axis and $z$-axis [4] directions shown in Figure 2 conforms to the elliptic equation.

$$
\left(\frac{S_{z}}{A_{0}}\right)^{2}+\left(\frac{S_{x}}{\left(k A_{0} h / 2\right)}\right)^{2}=1 .
$$

From formula (4), the expression of the vibration velocity of the stator torus particle along the transverse direction ( $z$-axis direction in Figure 2$)$ is shown as 


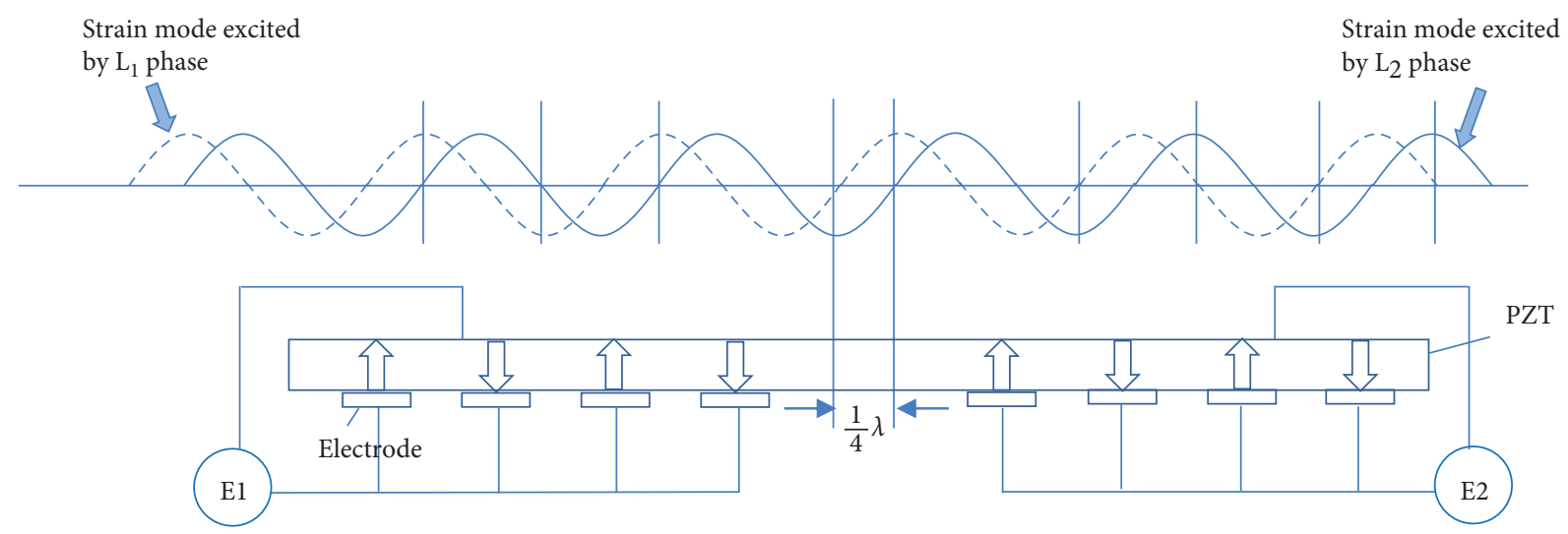

FIGURE 1: The traveling wave formed by the superposition of two standing waves of surface particle vibration after stator outspread $(\lambda$ : wavelength).

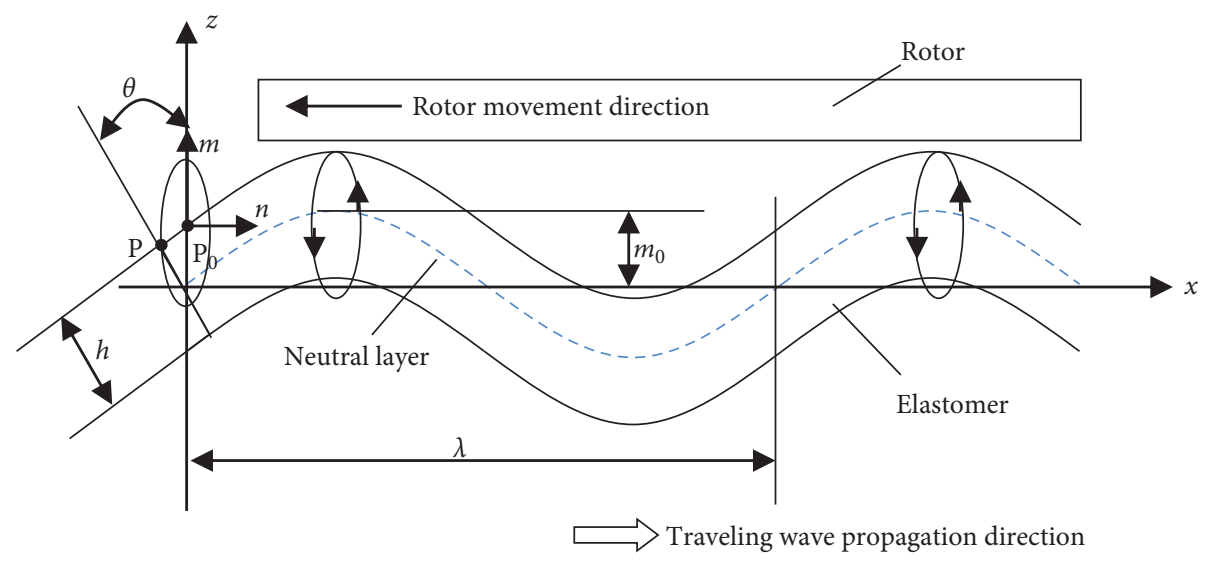

FIgURE 2: Principle of stator particle vibration.

$$
v_{s z}=\frac{\partial S_{z}}{\partial t}=-\omega A_{0} \sin (k x-\omega t) .
$$

From formula (5), the expression of the vibration velocity of the stator torus particle along the longitudinal direction ( $x$-axis direction in Figure 2 ) is shown as

$$
v_{s x}=\frac{\partial S_{x}}{\partial t}=-\frac{1}{2} k h \omega A_{0} \cos (k x-\omega t) .
$$

The negative sign in the formula indicates that the moving direction of the particle on the stator torus is opposite to the forward direction of the traveling wave.

2.2.2. Mathematical Model of Rotor Speed. According to formula (6), when the particle on the stator surface vibrates to the wave peak point $x_{p}$, the vibration displacement $S_{z}$ in the $z$-axis direction reaches the maximum, and the vibration displacement in the $x$-axis direction is zero. According to formulas (6) and (8), the tangential velocity when the stator particle is at the wave peak point $x_{p}$ is

$$
v_{x p}=\frac{-k \omega A_{0} h}{2}
$$

By ignoring the sliding phenomenon between the stator and the rotor [6], it is assumed that the contact surface between the stator and the rotor is just tangent [7] to the vibration waveform of the particle on the stator surface. The rotor speed is equal to the tangential speed when the stator particle is at the wave peak $x_{p}$, shown as

$$
v_{r}=v_{x p}=\frac{-k \omega A_{0} h}{2} \text {. }
$$

2.2.3. Hertz Contact Model of the Stator and Rotor. Let $k x^{\prime}=$ $k x-\omega t$ (that is $\left.x^{\prime}=x-\omega t / k\right)$. Formulas (5) and (8) can be rewritten as follows:

$$
S_{x}=\frac{k h A_{0}}{2} \sin (k x-\omega t),
$$

$v_{s x}=\frac{\partial S x}{\partial t}=-\frac{1}{2} k h \omega A_{0} \cos (k x-\omega t)=-\frac{1}{2} k h \omega A_{0} \cos \left(k x^{\prime}\right)$.

According to the friction mechanism of stator and rotor contact surface, the distance $D_{R}$ expression between stator neutral layer and contact surface can be obtained, shown as

$$
D_{R}=A_{0} \cos \left(k x_{0}\right)+\frac{h}{2} \text {. }
$$

The letter $x_{0}$ in formula (13) represents the boundary point of the contact area. 


$$
x_{0}=\frac{1}{k} a \cos \left(\frac{D_{R}-(h / 2)}{A_{0}}\right) \text {. }
$$

The letter $a$ in formula (14) represents the hertz contact width [2].

When the stator and rotor are at constant speed, the following can be obtained from formula (12):

$$
\frac{1}{2} k h \omega A_{0} \cos \left(k x_{1}\right)=\omega_{r} R
$$

The letter $\omega_{r}$ in formula (15) represents rotor speed, and $R$ represents radius. The letter $x_{1}$ represents isokinetic point, whose expression is as follows:

$$
x_{1}=\frac{1}{k} a \cos \left(\frac{2 \omega_{r} R}{k h \omega A_{0}}\right) \text {. }
$$

In the contact area of positive and negative $x$, the coincidence height of stator and rotor in the axial direction is shown as

$$
\Delta h=A_{0}\left(\cos k x^{\prime}-\cos k x_{0}\right) .
$$

By assuming that the elastic coefficient of the contact surface is $C_{N}$, according to Hooke's law, the expression of the axial pressure on the contact surface per unit length can be obtained and shown as

$$
f(x)=c_{N} \Delta h=c_{N} A_{0}\left(\cos k x^{\prime}-\cos k x_{0}\right) .
$$

Set the sliding friction coefficient of rotor contact surface as $\mu$, According to Coulomb's law, the expression of driving friction (expressed by the letter $F_{R}$ ) generated by a single traveling wave can be described as

$$
\begin{aligned}
F_{R} & =2 \mu\left(\int_{0}^{x_{1}} f(x) \mathrm{d} x-\int_{x_{1}}^{x_{0}} f(x) \mathrm{d} x\right) \\
& =2 \mu c_{N} A_{0}\left[2\left(\frac{1}{k} \sin k x_{1}-x_{1} \cos k x_{0}\right)-\left(\frac{1}{k} \sin k x_{0}-x_{0} \cos k x_{0}\right)\right] .
\end{aligned}
$$

The expression of synthetic torque generated by $\mathrm{N}$ wavepeaks is obtained from (19) and described as

$$
T=N F_{R} R \text {. }
$$

The axial pressure (expressed by the letter $F_{Z}$ ) expression of the traveling wave peak in the stator to the rotor is obtained from (18) and described as

$$
F_{Z}=N \int_{-x_{0}}^{x_{0}} f(x) \mathrm{d} x=2 N c_{N} A_{0}\left(\frac{1}{k} \sin k x_{0}-x_{0} \cos k x_{0}\right) \text {. }
$$

The rotational motion equation of rotor is shown as

$$
J \dot{\omega}_{r}=N F_{R} R-T_{L}
$$

The letter $J$ represents the moment of inertia, and the letter $T_{L}$ represents the load torque in formula (22).

The expression of rotor axial motion equation is described as follows:

$$
m_{r} \ddot{\omega}_{r}+d_{Z} \dot{\omega}_{r}=F_{Z}-F_{N}
$$

The letter $m_{r}$ represents the rotor mass, the letter $d_{z}$ represents the axial damping coefficient, and the letter $F_{N}$ represents preload in formula (23).

According to formula (22), the direct driving force for the rotation of two-phase traveling wave ultrasonic motor is the friction (expressed by the letter $F_{R}$ ) generated by the contact interface between stator and rotor under the action of circumferential preload. It can be seen from formula (10) that the speed and direction of rotor rotation change with the transverse movement of stator surface particles at the traveling wave peak and the traveling wave traveling direction. It can be seen from formula (23) that the axial motion of two-phase traveling wave ultrasonic motor is realized by the axial thrust (expressed by the letter $F_{z}$ ) of elliptical motion of particles on the stator surface. From formulas (10), (22), and (23), the elliptical trajectory of stator particles can be adjusted by changing the frequency, amplitude, and phase difference of two-phase driving voltage, so as to change the speed of ultrasonic motor. That is, the frequency of the driving voltage determines the value of angular velocity (expressed by the letter $\omega$ ) in the previously mentioned formulas, and the amplitude and the phase difference (expressed by the letter $\alpha$ ) of driving voltage determine the size of $A_{0}, F_{R}$ and $F_{z}$ in the previously mentioned formulas. In order to simplify the derivation formula, the phase difference (expressed by the letter $\alpha$ ) is discussed in this paper, and both are set to the fixed value of 90 degrees, shown as formula (1).

In this section, the corresponding mathematical model is built according to the structural principle of ultrasonic motor, which provides a theoretical basis for the design of speed control scheme.

\section{Speed Stability Control Based on Double Feedback}

3.1. Speed Stability Control Strategy. It can be seen from the previously mentioned formula that the speed regulation of ultrasonic motor can be realized by adjusting the frequency amplitude and phase difference of driving voltage [8]. In this paper, the speed control of ultrasonic motor is realized in the form of double feedback of voltage signal and current signal. The design principle diagram is shown in Figure 3.

The purpose of double feedback is to realize the function of constant voltage and constant current through closedloop voltage and closed-loop current. The double feedback control in Figure 3 adopts the mode of current inner loop and voltage outer loop. The current in the inner loop feedback closely tracks the voltage waveform and lags behind the voltage in phase. Compared with the prediction, the current lag achieves AC synchronization and unity power factor. The purpose of the voltage outer loop is to compare the effective value of the sampled output voltage and the input voltage, so as to realize the flow direction of PWM control power and realize the two-way control of voltage. In the current stabilizing working state, the voltage loop exits, the current loop is adopted, and the output of the regulator 


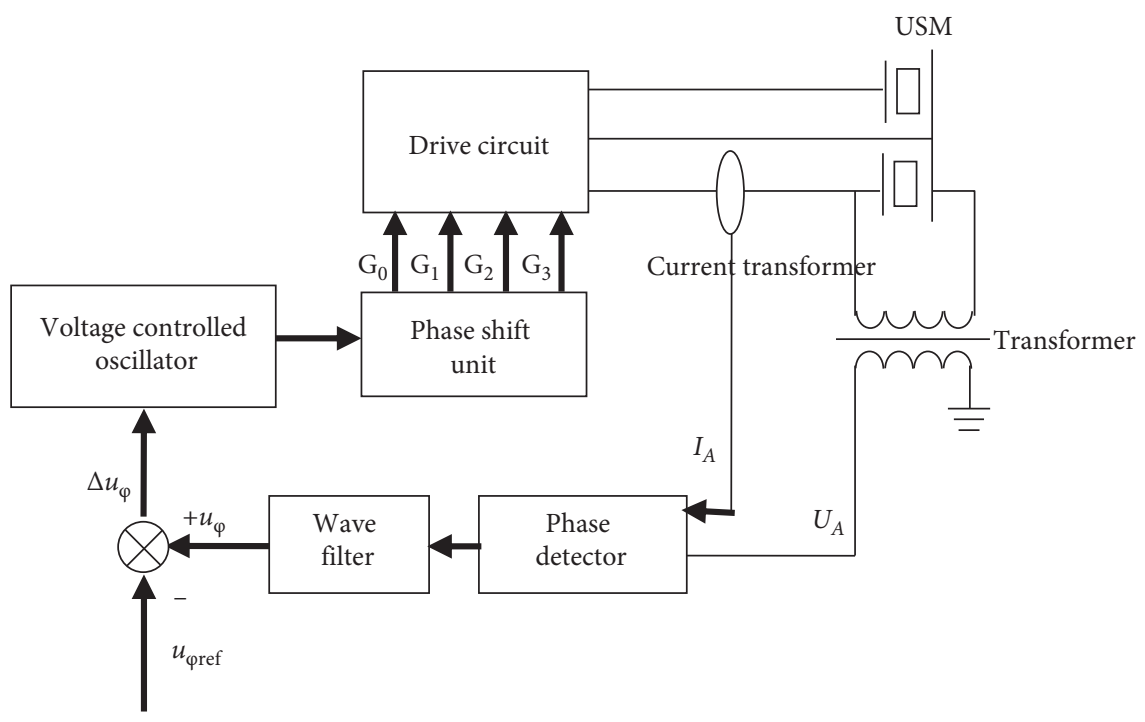

Figure 3: Speed control strategy.

of the current loop is assigned to the phase-shift unit to generate digital pulses to control the switching state of MOSFET to achieve the purpose of current stabilizing. According to formula (10), the rotor speed depends on amplitude $A_{0}$. It can be seen from formula (1) and the schematic diagram shown as Figures 1 and 2 that the amplitude $A_{0}$ depends on the voltage amplitude. Therefore, the rapid stability of voltage or current can ensure the rapid stability of speed.

In Figure 3, $u_{\varphi \mathrm{ref}}$ is set as the operating point of the motor. A voltage signal (described as $u_{\varphi}$ in Figure 3 ) can be obtained after the current (described as $I$ in Figure 3 ) and voltage (described as $U$ in Figure 3 ) through phase-detector and wave-filter, which reflects the impedance angle (described as $\varphi$ in Figure 3) of the motor at the current driving frequency. A deviation voltage signal (described as $\Delta u_{\varphi}$ in Figure 3 ) can be obtained by contrasting between $u_{\varphi}$ and

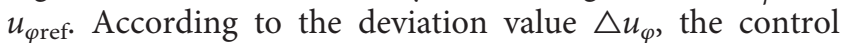
voltage of the voltage controlled oscillator (VCO) is changed, and the output frequency of VCO is adjusted. The control voltage and the output frequency of the voltage controlled oscillator can be adjusted according to the value of deviation voltage signal (described as $\Delta u_{\varphi}$ in Figure 3). According to the output frequency of the voltage controlled oscillator, the phase-shift unit generates a quadrature PWM waveform. The PWM wave drives the ultrasonic motor after boosting and power amplification by the driving circuit, so as to ensure the stability of the motor speed.

3.2. Driving Circuit of the Ultrasonic Motor. In order to realize the driving function of the driving circuit in Figure 3 for the two-phase traveling wave ultrasonic motor, the driving circuit shall meet the requirements of high output voltage frequency and large amplitude, and the output voltage frequency and amplitude shall be adjustable. Thus, the speed of ultrasonic motor can be controlled by adjusting the frequency and amplitude of driving voltage.
If the frequency and amplitude of the two-phase driving voltage of the ultrasonic motor are equal and adjustable, respectively, and the two-phase voltage is orthogonal and the driving voltage frequency matches the stator resonant frequency, the two single-phase standing waves can synthesize the traveling waves on the piezoelectric ceramic surface. In this paper, two-phase H-bridge [9] circuit is used to drive two-phase traveling wave ultrasonic motor. The two-phase $\mathrm{H}$-bridge adopts four MOSFET switches, and the on-off of each switch is controlled by the phase-shift PWM signal generation unit. The DC voltage is converted into highfrequency square wave voltage. By passing through the transformer and series matching inductance, the square wave voltage can drive two-phase piezoelectric ceramic sheets of ultrasonic motor respectively after resonant amplification and filtering. After the piezoelectric ceramic sheet is successfully driven, the two-phase standing wave meeting certain conditions is obtained, and the traveling wave is synthesized to drive the rotor to rotate. The series matching inductance is used to filter out the high-frequency harmonic components in the square wave voltage. The schematic diagram is shown in Figure 4.

In Figure 4, M1-M8 is 8 MOSFET switches, and the onoff of each tube is controlled by the corresponding PWM1-PWM8 signal. M1-M4 constitutes H-bridge of phase A, and M5-M8 constitutes $\mathrm{H}$-bridge of phase $\mathrm{B}$. The duty cycle of the PWM signal of M1-M8 is a fixed value of $50 \%$ (excluding dead band), and the PWM signals of the upper and lower bridge arms are complementary.

3.3. Phase Shift PWM Generating Unit. The phase-shift [3] unit in Figure 3 adopts phase-shift PWM control and outputs PWM signal to the driving circuit (Figure 4) to control the on-off state of M1-M8, so that the output voltage waveform of the $\mathrm{H}$-bridge of phase $\mathrm{A}$ and $\mathrm{H}$-bridge of phase $\mathrm{B}$ in Figure 4 is only determined by the PWM control signal, and the output voltage is independent of the load nature and 


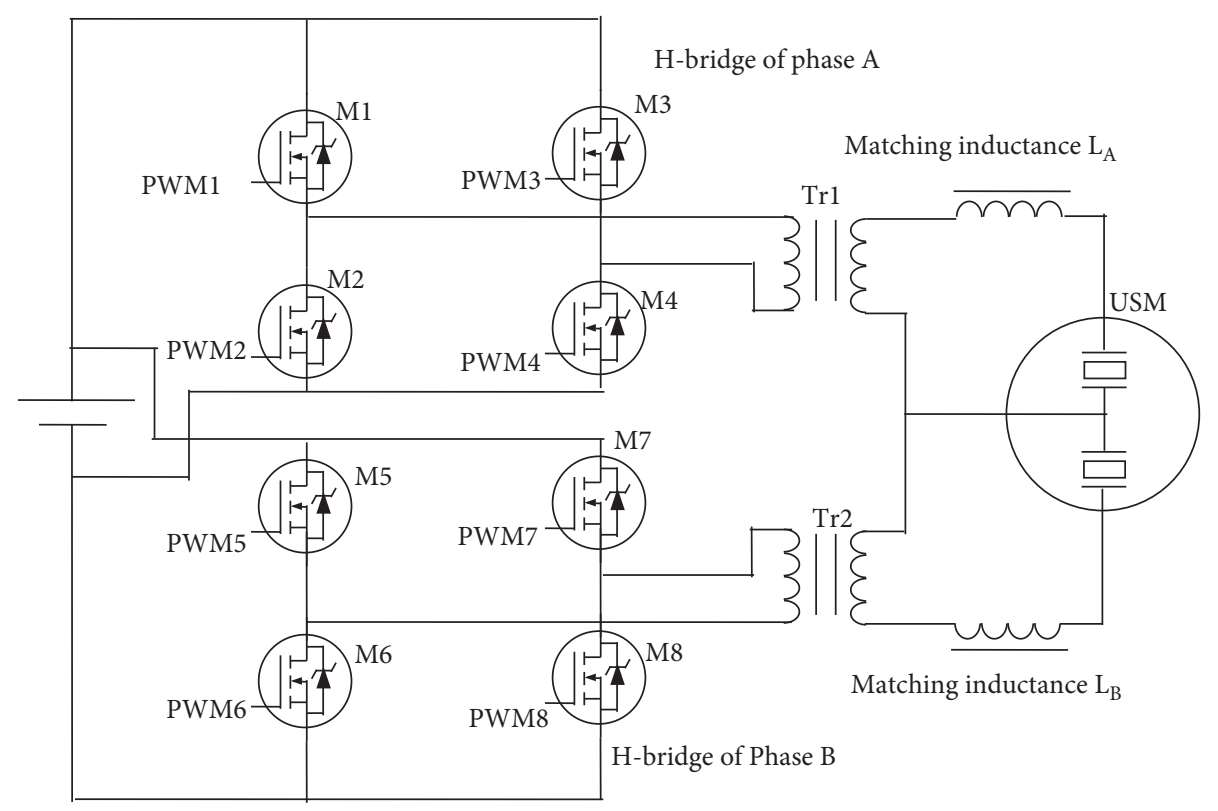

FIGURE 4: USM driving circuit based on H-bridge.

freewheeling process. The output voltage drives the ultrasonic motor after through transformer $\operatorname{Tr} 1$ (or $\operatorname{Tr} 2$ ) and matching inductance $L 1$ (or L2) in Figure 4.

The schematic diagram of phase-shift PWM unit is shown as Figure 5, which outputs eight channels of phaseshift PMM control signals PWM1-PWM8 to control the onoff state of two-phase H-bridge M1-M8 in Figure 4. This made the frequency, amplitude, and phase difference of the output voltage of $\mathrm{H}$-bridge of phase $\mathrm{A}$ and $\mathrm{H}$-bridge of phase $\mathrm{B}$ able to continuously adjust, so as to meet the requirements of the two-phase ultrasonic motor for the output driving signal of the driving circuit and achieve the purpose of adjustable speed.

In view of the requirements of the driving link of ultrasonic motor, this section designs the driving circuit based on two-phase H-bridge and phase-shift PWM generating unit. According to the mathematical model of ultrasonic motor, the control scheme of rotor speed can be stabilized quickly based on voltage and current double feedback. The design scheme is reasonable and will be verified by simulation in the next section.

\section{Simulation Verification}

In order to verify the rationality of the previously mentioned speed control design, Matlab/Simulink software is used for simulation verification in this paper.

4.1. Simulation of Phase Shift PWM. According to Figure 5, the simulation model of phase-shift PWM control signal generator is built by using Matlab/Simulink software, as shown in Figure 6. The obtained output phase-shift PWM control signal is shown in Figure 7.

The waveforms of PWMA1 and PWMA2 shown in Figure 7 are the output waveforms of output signals $A 1$ and $A 2$ in Figure 6, which corresponds to PWM1 and PWM2 in
Figure 5 and meets the complementarity of upper and lower bridge arm switching devices.

4.2. Simulation of the Driving Circuit. According to Figure 4, the simulation model of the driving circuit of the ultrasonic motor is built, as shown in Figure 8. The voltage amplitude is set as $130 \mathrm{v}$, the frequency is set as $40.5 \mathrm{kHz}$, and the duty cycle is set as 0.5 . The driving voltage waveform from the two-phase H-bridge is obtained from Figure 8, as shown in Figure 9.

The phase difference (expressed by the letter $\alpha$ ) of the output voltage waveform can be read out from Figure 9 is 90 degrees, which meets the setting conditions of formula (1).

4.3. Simulation of Stator Particle Vibration. The simulation module of piezoelectric ceramics and stator particle vibration is built according to formulas (13)-(23), shown as Figure 10, and the parameter settings during simulation are shown in Table 1.

The input signal in Figure 10 is the output signal in Figure 8, that is, $\mathrm{u}-\mathrm{cp} 1$ and $\mathrm{u}-\mathrm{cp} 2$ shown as Figure 9. The stator two-phase vibration waveform can be obtained, as shown in Figure 11.

The variation trend of traveling wave vibration waveform synthesized by superposition of two-phase standing waves is obtained from the two-phase vibration waveform of stator in Figure 11, which is shown in Figures 12 and 13. The amplitude variation waveform of traveling wave is shown in Figure 12, and the amplitude waveform of traveling wave obtained by Fourier transform is shown in Figure 13.

The given frequency $f$ is $40.5 \mathrm{kHz}$ and the voltage amplitude is $130 \mathrm{v}$ in the simulation process of Figures 6-13. According to the conclusions of formulas (10), (22), and (23), the different vibration effects of stator surface particles can be obtained by changing the amplitude, frequency, and 


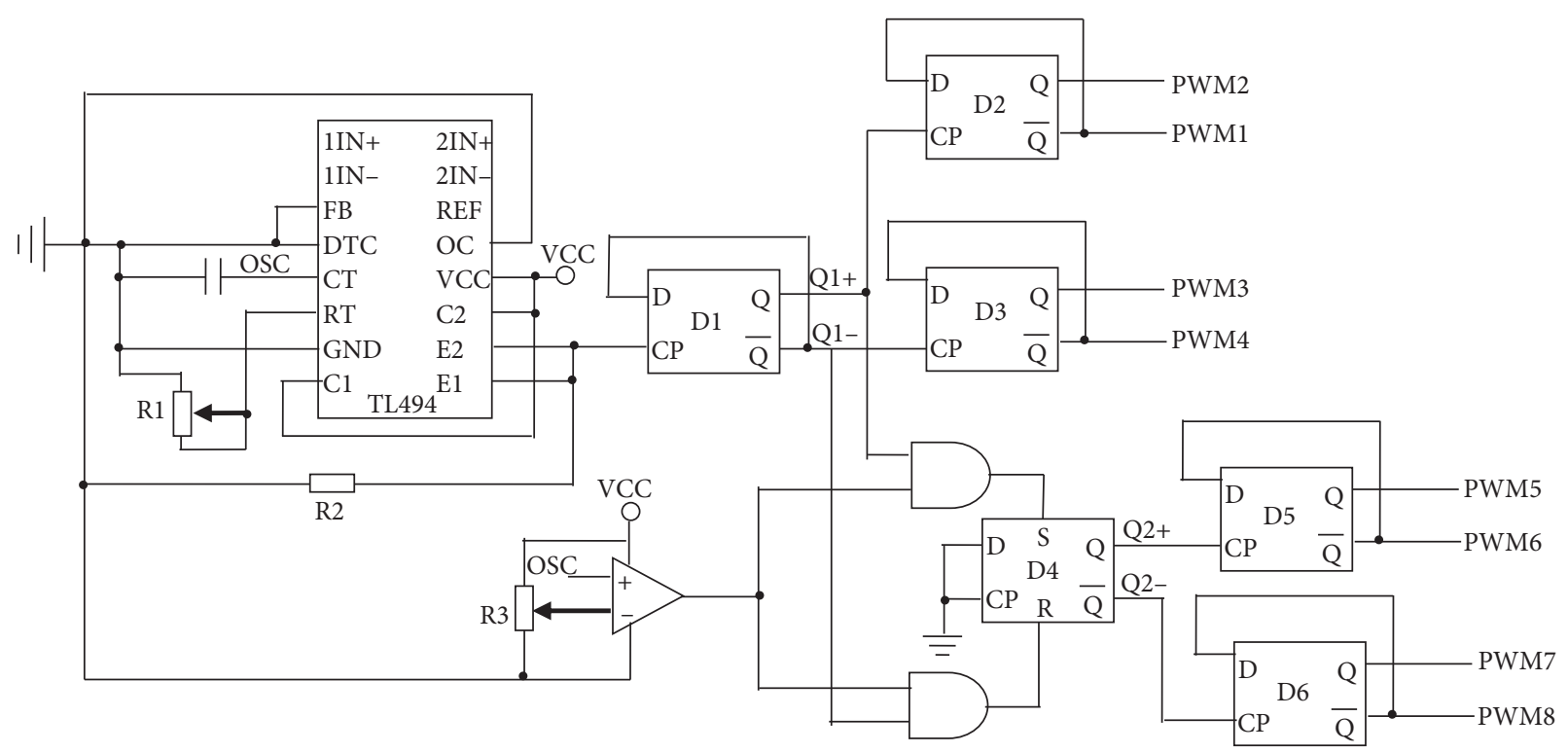

FIgure 5: Phase-shift PWM unit.

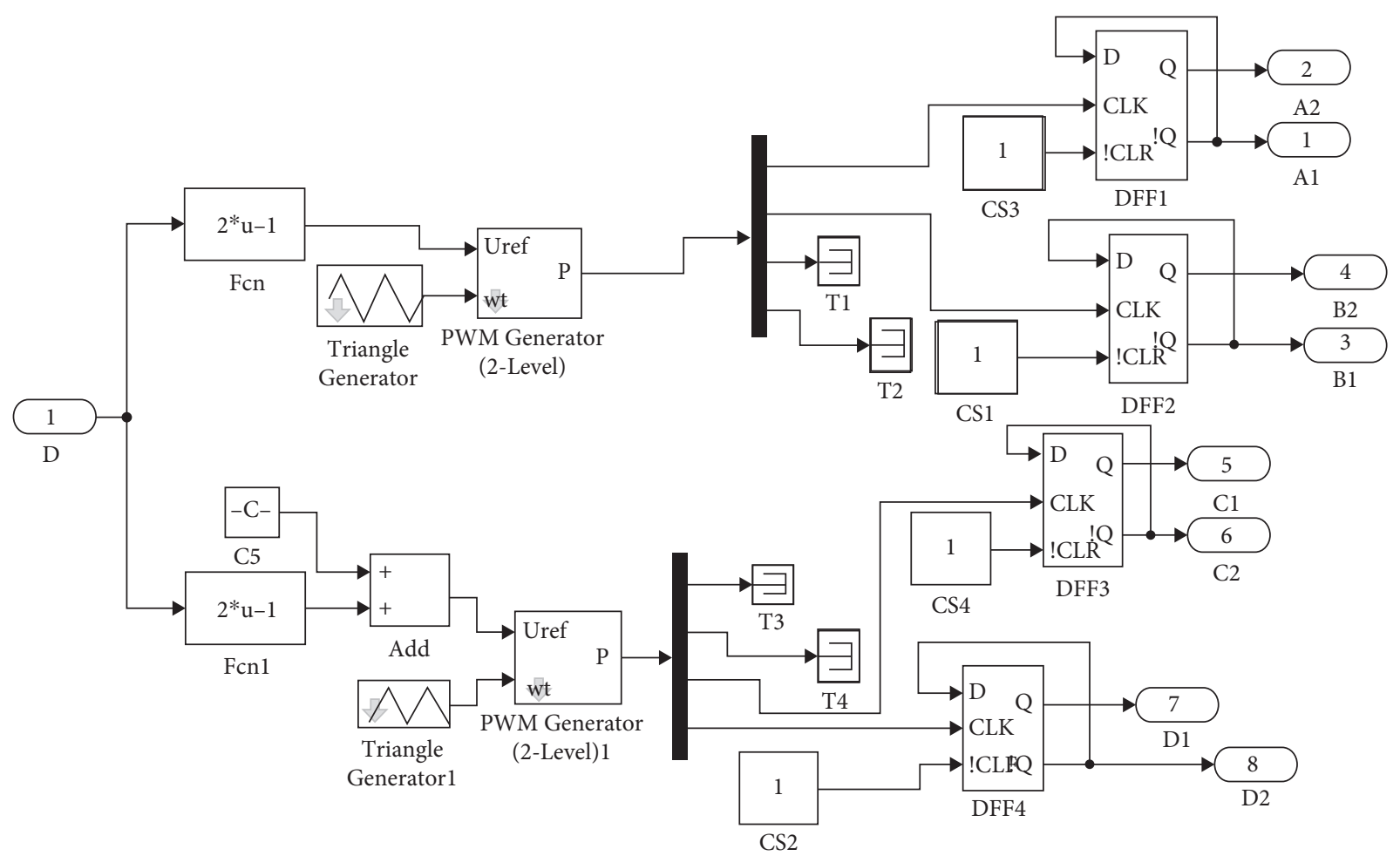

FIgURE 6: Phase-shift PWM generator simulation module.

phase difference of two-phase driving voltage. The simulation results are shown in Table 2 . In this paper, the phase difference (expressed by the letter $\alpha$ ) is the fixed value, which is 90 degrees.

It can be seen from Table 2 that when the driving voltage amplitude is fixed at $200 \mathrm{~V}$, the frequency (expressed by the letter $f$ ) changes and increases from $34.5 \mathrm{kHz}$ to $41.5 \mathrm{kHz}$, and the amplitude will reach the maximum value when the frequency is $33.5 \mathrm{kHz}$. And it can be concluded that there is a nonlinear relationship between frequency and traveling wave vibration amplitude.

It can be seen from Table 2 that when the given frequency is $41.5 \mathrm{kHz}$ and the driving voltage amplitude is changed from $200 \mathrm{~V}$ to $110 \mathrm{~V}$, the vibration amplitude decreases gradually, and the relationship between vibration amplitude and driving voltage amplitude is almost linear, which is consistent with the derivation process of the previous formulas. 

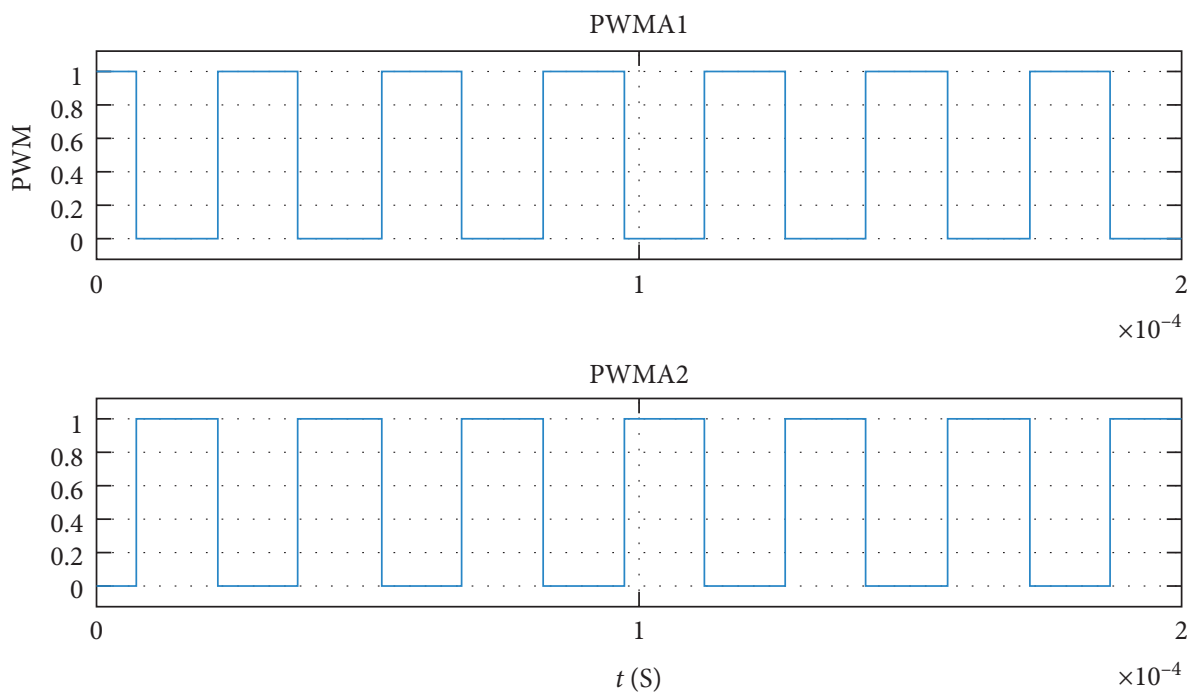

Figure 7: Corresponding PWM waveform.

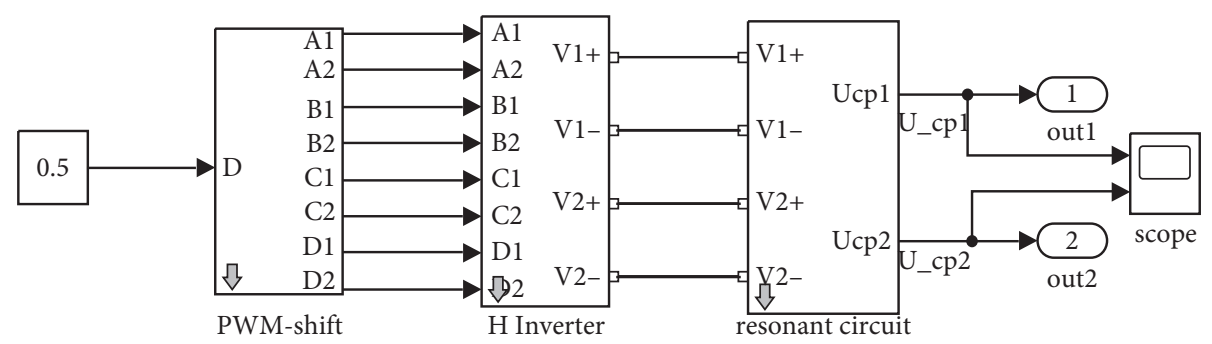

Figure 8: Simulation model of the driving circuit.

4.4. Simulation Verification of Rotor Speed. According to formulas (19)-(23), the stator and rotor contact simulation module is built, as shown in Figure 14, and the parameters in the simulation are shown in Table 3.

In Figure 14, the model input signal includes the response frequency of stator particle vibration and the amplitude of traveling wave vibration, and the output signal is the rotor speed. When the preload $F_{N}$ and load torque $T_{L}$ (no-load) are constant, the speed is simulated and calculated from small to large by using the traveling wave vibration amplitude data in Table 2 to obtain the speed of the rotor under different traveling wave amplitudes as shown in $\mathrm{Ta}$ ble 4 . The simulation calculation of rotating speed is carried out by using the traveling wave vibration amplitude data in Table 2 . The traveling wave vibration amplitude ranges from large to small, and the rotor speed under different traveling wave amplitudes is obtained as shown in Table 4.

According to Table 4, the broken line diagram shown in Figure 15 is obtained by using office tools. From the change trend of broken line in Figure 15, it is shown that the rotor speed of ultrasonic motor is almost linear with the amplitude of traveling wave, which is consistent with the previous analysis results and the conclusions of formulas (10) and (15).

Integrate the simulation modules Figures 6, 8, 10, and 14 into the system control simulation module, and replace the constant module in Figure 8 with the step signal generation module. When the step time is set to $[0,0.005,0.010,0.015]$, the amplitude is set to $[0.50,0.55,0.60,0.65]$, and the driving frequency (expressed by the letter $f$ ) is set to a fixed value of $41.5 \mathrm{kHz}$, the corresponding speed change waveform can be obtained, as shown in Figure 16.

It can be seen from Figure 16 that the rotor speed of twophase traveling wave ultrasonic motor can quickly stabilize after the step change of traveling wave amplitude. For example, after the amplitude step change of 0.05 occurs at $0.005 \mathrm{~s}$, the rotor speed can be stable within about $0.001 \mathrm{~s}$; after the amplitude step change of 0.05 occurs at $0.01 \mathrm{~s}$, the rotor speed can be stable within about $0.001 \mathrm{~s}$; after the amplitude step change of 0.05 occurs at $0.015 \mathrm{~s}$, the rotor speed can be stable within about $0.001 \mathrm{~s}$. It shows that the speed stability control strategy based on double feedback designed in this paper is good and can quickly stabilize the rotor speed. In Figure 16, the amplitude is large and the speed is high. It can be seen from Figure 16 that the variation trend of speed and amplitude meets the conclusion of broken line Figure 15 and formula (10); that is, the rotor speed has a linear relationship with the amplitude of traveling wave.

In this section, the previously mentioned design scheme is simulated and verified by simulation tools. The output waveform of each physical quantity in the simulation results meets the relationship between each physical quantity in the actual physical model of ultrasonic motor. In the voltage 


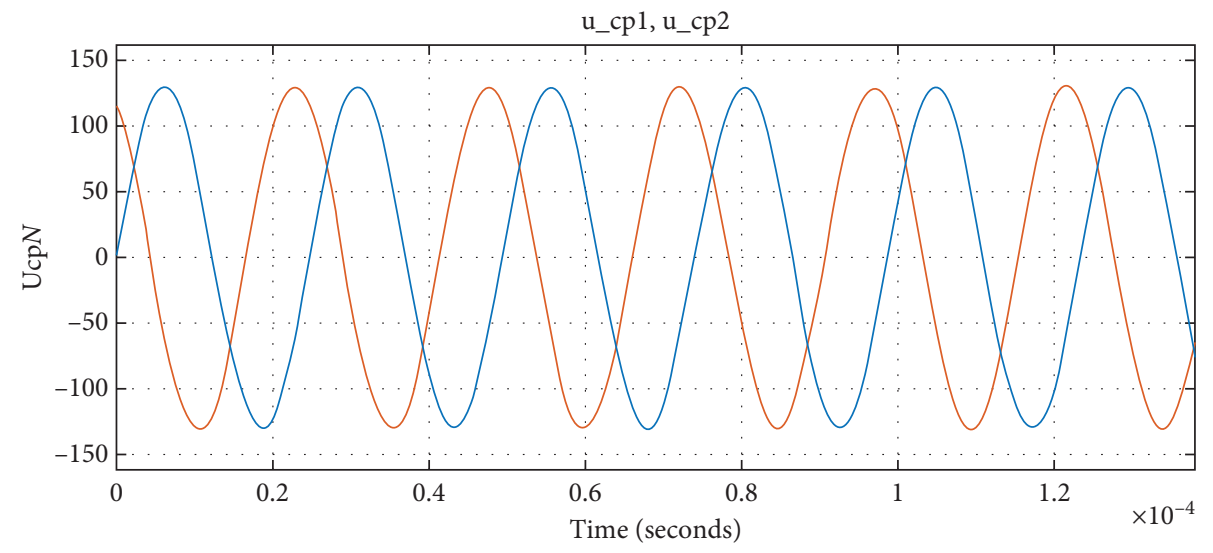

Figure 9: The driving voltage waveform of the two-phase H-bridge.

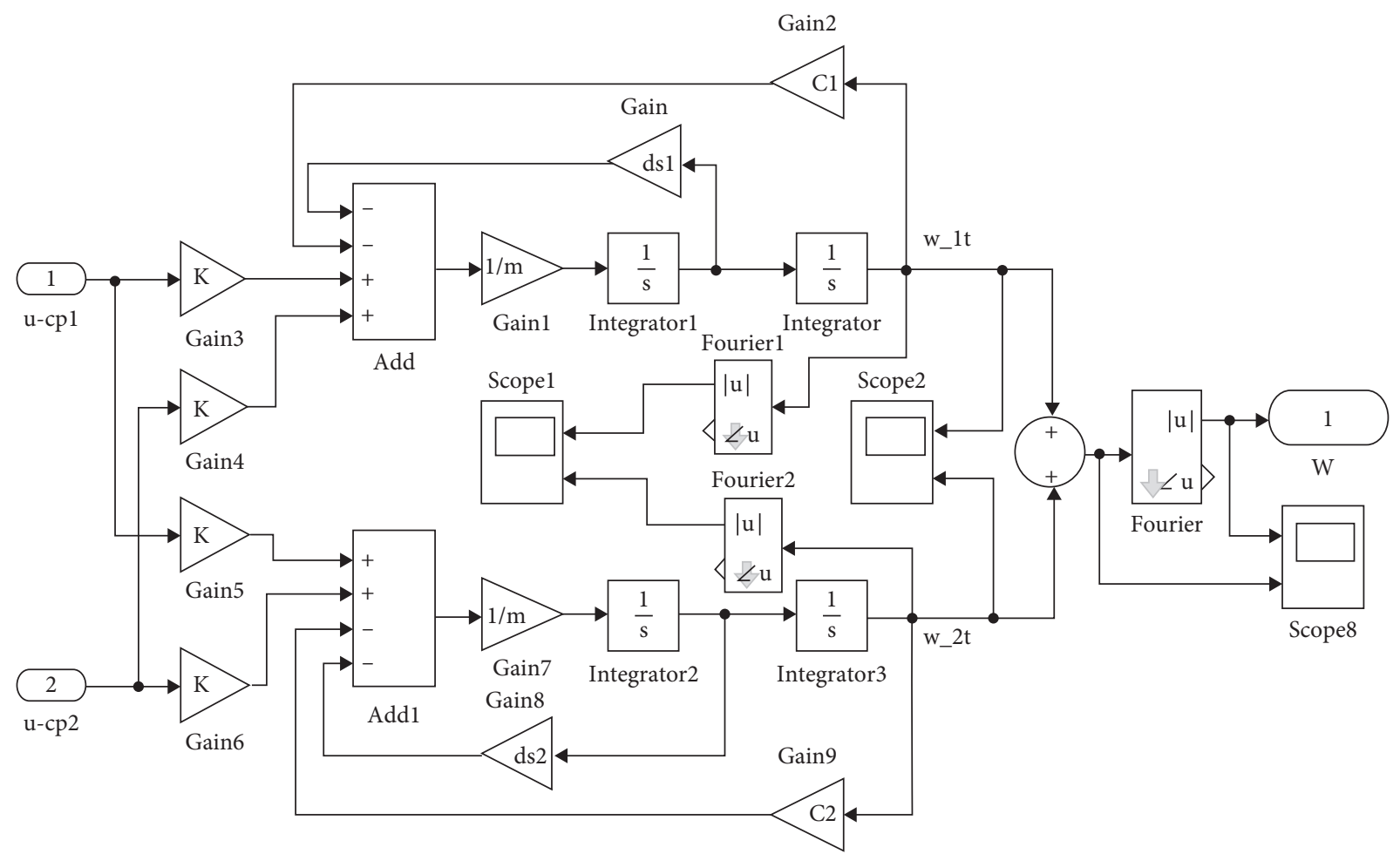

Figure 10: The simulation module of stator particle vibration.

TABLE 1: Vibration particle parameters.

\begin{tabular}{|c|c|c|c|c|c|}
\hline Name & Meaning & Value & Name & Meaning & Value \\
\hline$m$ & Equivalent mass & $0.112 \mathrm{~kg}$ & $A$ & Stiffness coefficient & $1.76 \times 10^{-10}$ \\
\hline$d_{s}$ & Damping coefficient & $580.8 \mathrm{Ns} / \mathrm{m}$ & $c_{1}$ & Modal hardness & $5.0371 \times 10^{9}$ \\
\hline$e$ & Asymmetry factor & 0.02 & $c_{2}$ & Modal hardness & $4.991 \times 10^{9}$ \\
\hline$c_{c}$ & Elastic coefficient of PZT & $2.3589 \times 10^{9}$ & & & \\
\hline
\end{tabular}




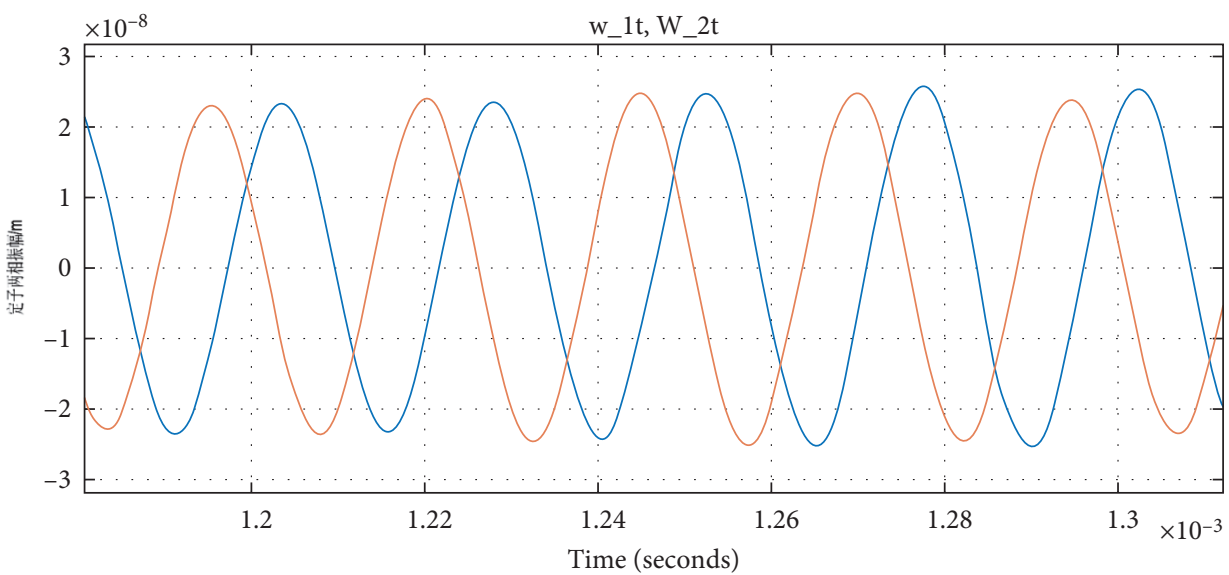

- w_1t

- W_2t

FIGURE 11: Stator two-phase vibration waveform.

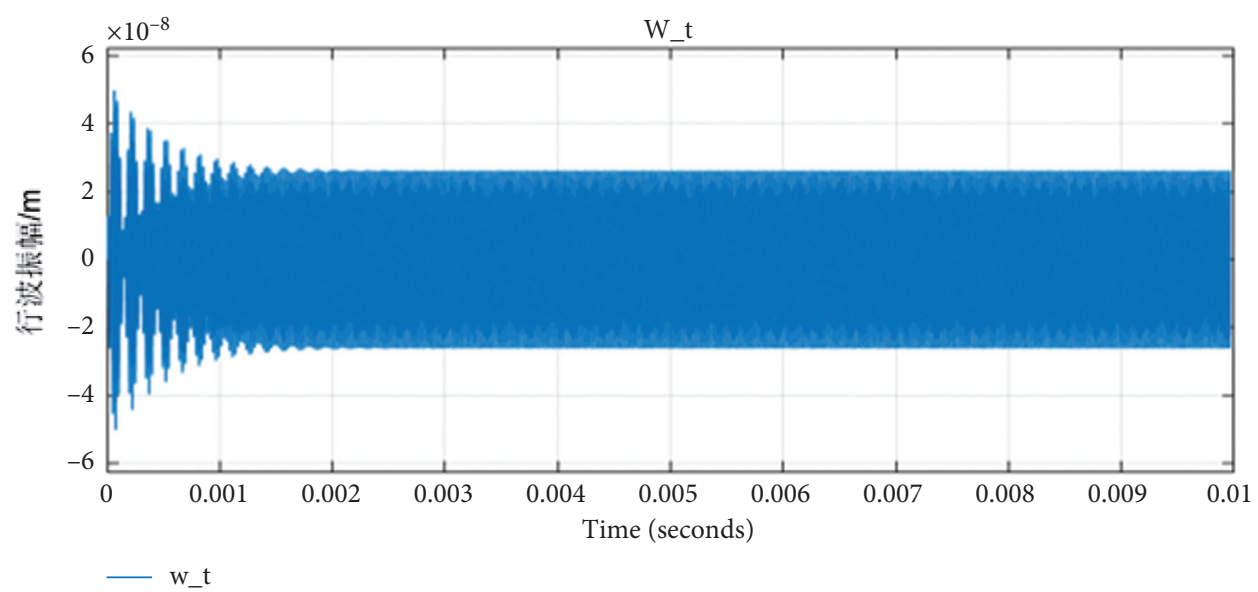

Figure 12: Amplitude waveform of traveling wave.

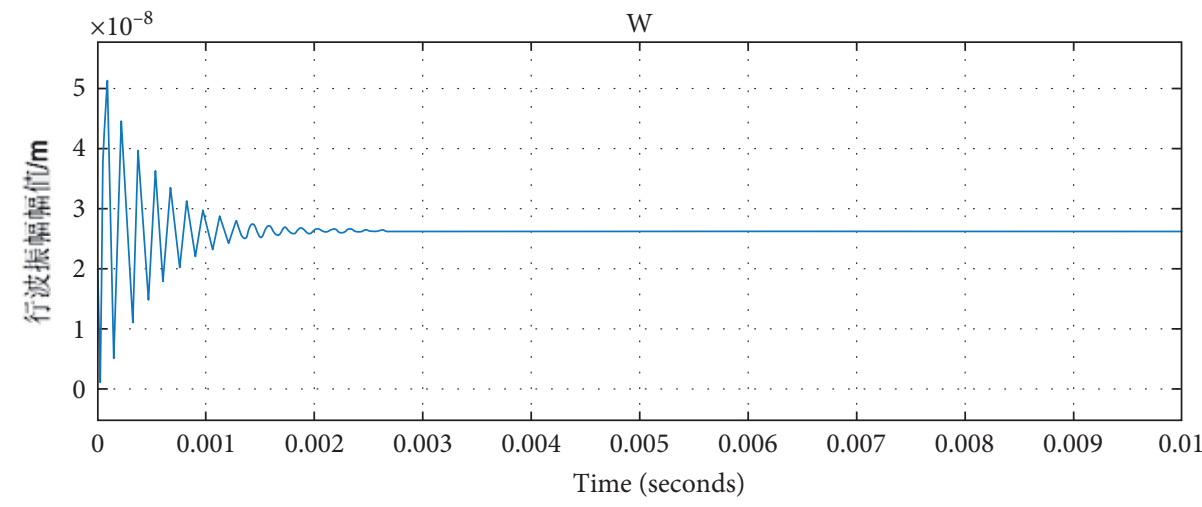

$-\mathrm{W}$

FIGURE 13: The vibration waveform of synthetic traveling wave. 
TABLE 2: Simulation results of traveling wave amplitude varying with driving voltage and frequency.

\begin{tabular}{lccccc}
\hline$U / \mathrm{V}$ & $f / \mathrm{kHz}$ & $\mathrm{AMP} / \mathrm{m}$ & $\mathrm{U} / \mathrm{V}$ & $f / \mathrm{kHz}$ & $\mathrm{AMP} / \mathrm{m}$ \\
\hline 200 & 34.5 & $3.91 \times 10^{-7}$ & 170 & 41.5 & $2.95 \times 10^{-8}$ \\
200 & 33.5 & $7 \times 10^{-7}$ & 140 & 41.5 & $2.44 \times 10^{-8}$ \\
200 & 38.5 & $5.91 \times 10^{-8}$ & 110 & 41.5 & $1.92 \times 10^{-8}$ \\
200 & 41.5 & $3.47 \times 10^{-8}$ & & & \\
\hline
\end{tabular}

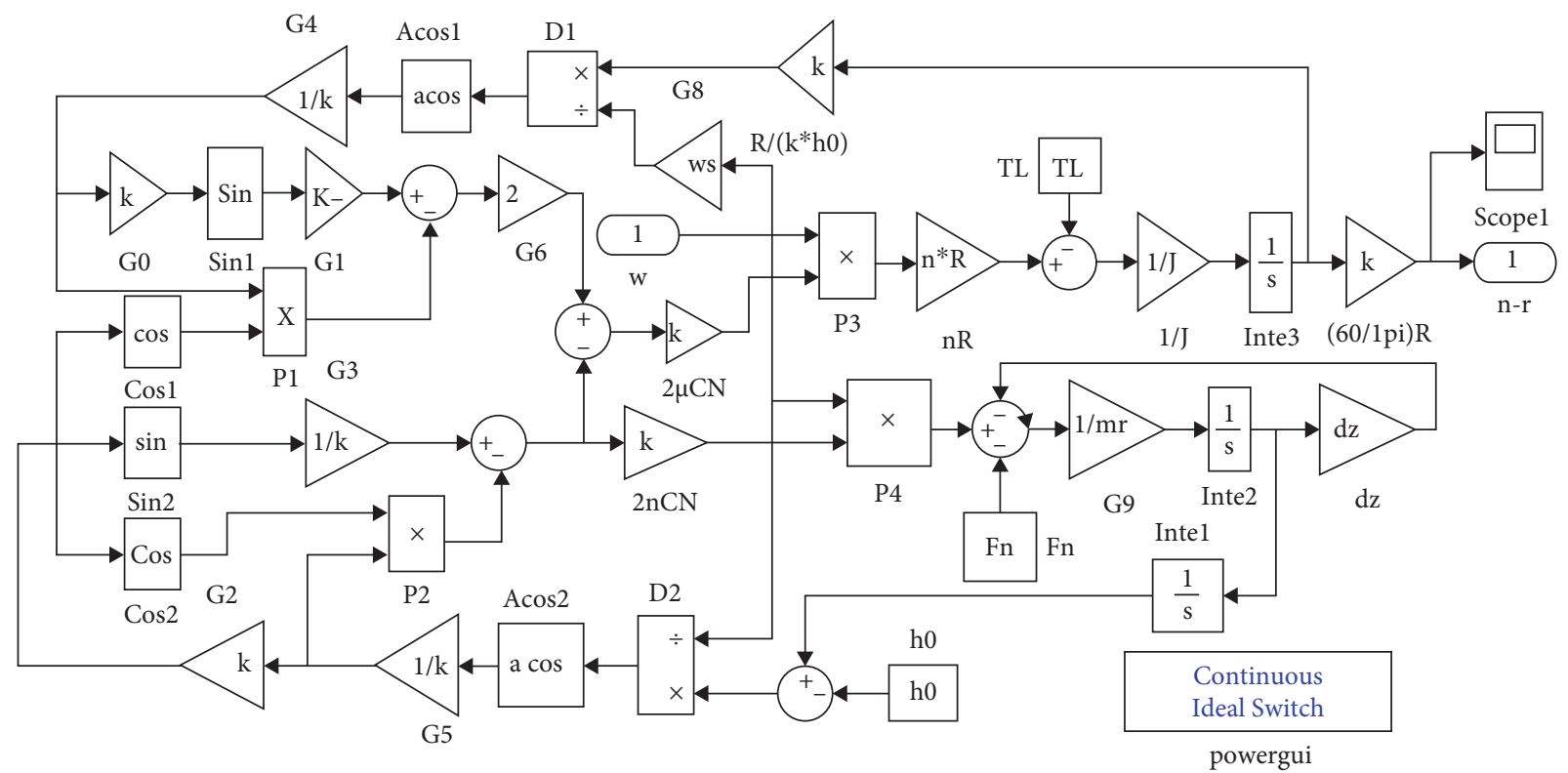

FIGURE 14: Stator rotor contact simulation model.

TABle 3: Parameters of the stator rotor contact simulation model.

\begin{tabular}{lccccc}
\hline Name & Meaning & Value & Name & Meaning & Value \\
\hline$n$ & Modal orders & 9 & $\lambda$ & Wavelength & $18.29 \times 10^{-3} \mathrm{~m}$ \\
$\mu$ & Friction coefficient & 0.1501 & $k$ & Wave numbers & $2 \pi / \lambda$ \\
$m_{r}$ & Rotor equivalent mass & $0.00811 \mathrm{~kg}$ & $J$ & Moment of inertia & $3.5 \times 10^{-6} \mathrm{~kg} \bullet \mathrm{m}^{2}$ \\
$c_{N}$ & Equivalent elasticity & $2.6 \times 10^{9} \mathrm{~N} / \mathrm{m}^{2}$ & $d_{z}$ & Damping coefficient & 15000 \\
$h_{0}$ & Half of equivalent thickness & $7 \times 10^{-3} \mathrm{~m}$ & $F_{N}$ & Rotor preload & $200 \mathrm{~N}$ \\
$R$ & Equivalent radius of rotor & $26.62 \times 10^{-3} \mathrm{~m}$ & $T_{L}$ & Load torque & $0 \mathrm{~N} \bullet \mathrm{m}$ \\
\hline
\end{tabular}

TABLE 4: Rotor speed varying with traveling wave vibration amplitude.

\begin{tabular}{lccc}
\hline AMP $/ \mathrm{m}$ & Speed $(\mathrm{r} / \mathrm{min})$ & AMP $(\mathrm{m})$ & Speed $(\mathrm{r} / \mathrm{min})$ \\
\hline $5.91 \times 10^{-8}$ & 238 & $2.44 \times 10^{-8}$ & 98 \\
$3.47 \times 10^{-8}$ & 139 & $1.92 \times 10^{-8}$ & 77 \\
$2.95 \times 10^{-8}$ & 119 & & \\
\hline
\end{tabular}

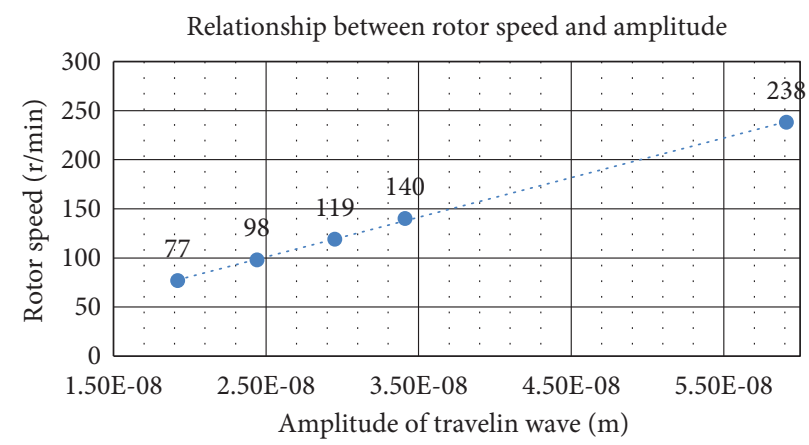

FIgURE 15: Broken line diagram of relationship between amplitude and speed. 


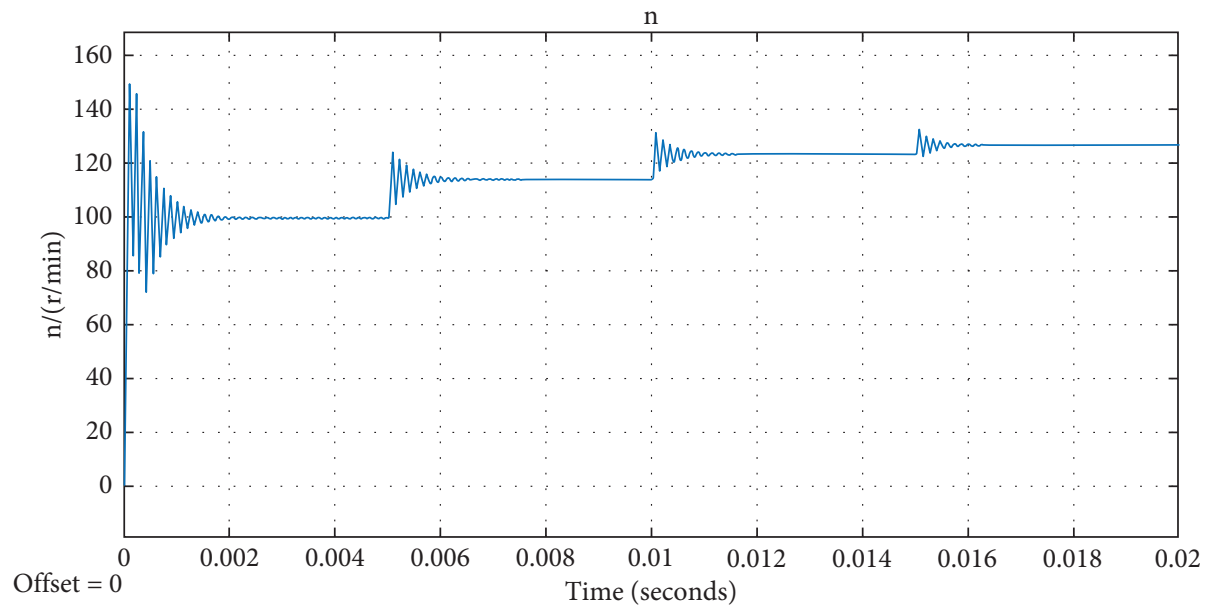

FiguRE 16: Speed change waveform.

current double feedback control mode, the simulation waveform of rotor speed can also be quickly stabilized in a short time, which achieves the design goal of this paper.

\section{Conclusion}

Combined with the two-phase H-bridge control circuit of phase-shift PWM, a speed stability control strategy based on double feedback is designed in this paper. The simulation verification is carried out by using Matlab/Simulink simulation tool. The variation trend of each physical quantity in the simulation results is consistent with the actual physical model. The function that the rotor speed can be quickly stabilized of two-phase traveling wave ultrasonic motor is realized.

\section{Data Availability}

All data generated or analyzed during this study are included within this article.

\section{Conflicts of Interest}

The authors declare that they have no conflicts of interest.

\section{References}

[1] H. Zhang, "Design and implementation of ultrasonic motor compound drive control system," vol. 6, Harbin Institute of Technology, Harbin, China, 2018, Master's Thesis.

[2] M. Brahim, I. Bahri, and Y. Bernard, "Real time implementation of $\mathrm{H}$-infinity and RST motion control of rotary traveling wave ultrasonic motor," Mechatronics, vol. 44, pp. 14-23, 2017.

[3] F. R. Mohd Romlay, W. A. Wan Yusoff, and K. A. Mat Piah, "Increasing the efficiency of traveling wave ultrasonic motor by modifying the stator geometry," Ultrasonics, vol. 64, pp. 177185, 2016.

[4] S. Pan, J. Zhang, W. Huang, and S. Yunlai, "Butterfly-shaped linear ultrasonic motor robust control based on Takagi-Sugeno fuzzy systems," Guangxue Jingmi Gongcheng/Optics \& Precision Engineering, vol. 22, no. 10, pp. 7817-7821, 2014.
[5] Y.-C. Liu, S. Laghrouche, D. Depernet, A. Djerdir, and M. Cirrincione, "Disturbance-observer-based complementary sliding-mode speed control for PMSM drives: a super-twisting sliding-mode observer-based approach," IEEE Journal of Emerging and Selected Topics in Power Electronics, vol. 9, no. 5, pp. 5416-5428, 2021.

[6] Y. Liu Qiang, "The Research of Investigating the modle of USM and its Driving system," vol. 5, Wuhan University of Technology, Wuhan, China, 2013, Master's Thesis.

[7] J. Shi and F. Lv, "Self-tuning nonlinear generalized predictive speed control of ultrasonic motors," Proceedings of the CSEE, vol. 32, no. 27, pp. 66-72, 2021.

[8] A. Gencer, "A comparative speed/position control technique based fuzzy logic control for travelling wave ultrasonic motor," in Proceedings of the 2015 7th International Conference on Electronics, Computers and Artificial Intelligence (ECAI), Bucharest, Romania, June 2015.

[9] S. Fujimoto, T. Namihira, K. Iwata, and M. Kobayashi, "Curie temperature and high temperature behavior of $\mathrm{Pb}(\mathrm{Zr}, \mathrm{Ti}) \mathrm{O}_{3} /$ $\mathrm{Pb}(\mathrm{Zr}, \mathrm{Ti}) \mathrm{O}_{3}$ sol-gel composites," Japanese Journal of Applied Physics, vol. 54, no. 7, pp. 2395-3470, 2015. 